\title{
Corela
}

Cognition, représentation, langage

HS-8 | 2010

L'interpellation

\section{Interpellation et violence verbale : essai de typologisation}

Nathalie Auger, Béatrice Fracchiolla, Claudine Moïse et Christina Schultz-Romain

\section{OpenEdition}

Journals

Édition électronique

URL : http://journals.openedition.org/corela/1023

DOI : 10.4000/corela.1023

ISSN : $1638-573 X$

\section{Éditeur}

Cercle linguistique du Centre et de l'Ouest - CerLICO

\section{Référence électronique}

Nathalie Auger, Béatrice Fracchiolla, Claudine Moïse et Christina Schultz-Romain, «Interpellation et violence verbale : essai de typologisation », Corela [En ligne], HS-8 | 2010, mis en ligne le 23 novembre 2010, consulté le 19 avril 2019. URL : http://journals.openedition.org/corela/1023 ; DOI : 10.4000/ corela.1023

Ce document a été généré automatiquement le 19 avril 2019

\section{(c) (i) (2)}

Corela - cognition, représentation, langage est mis à disposition selon les termes de la licence Creative Commons Attribution - Pas d'Utilisation Commerciale - Partage dans les Mêmes Conditions 4.0 International. 


\title{
Interpellation et violence verbale : essai de typologisation
}

\author{
Nathalie Auger, Béatrice Fracchiolla, Claudine Moïse et Christina \\ Schultz-Romain
}

Merci à Britta Langhans pour la relecture attentive de cet article.

Dans le cadre de notre recherche, nous nous proposons d'analyser l'interpellation à partir de différents terrains (espaces publics - rue, transports en commun -, établissements scolaires, médias etc.). Telle que décrite, la violence verbale représente

une montée en tension contextualisée qui se décline à travers différentes étapes (incompréhension, négociation, évitement, renchérissement, renforcement...), marquée par des " déclencheurs de conflit » et par l'emploi d'une importante variété d'actes de langage (harcèlement, mépris, déni, insulte...) (Auger et Moïse, 2005).

Dans différents articles, nous avons donc défini la violence verbale comme un processus complexe, situationnel et interactionnel. C'est dans cette perspective que nous envisageons l'étude de l'interpellation qui constitue souvent, en effet, un acte de langage fondateur et originel, déclencheur de la montée en tension. Nous essayons de voir quelles sont les caractéristiques linguistiques de l'interpellation dans un processus de violence verbale, et quelles formes elle peut revêtir. Nous analysons aussi, à partir d'exemples, dans quels types de contextes elle est susceptible de déclencher de la violence verbale.

\section{Valeurs de l'interpellation pour une analyse de la violence verbale}

3 L'interpellation peut prendre toutes les différentes formes linguistiques que sont les termes d'adresse. Ces termes d'adresse correspondent à l'ensemble des expressions dont dispose le locuteur pour désigner son ou ses allocutaires (André-Larochebouvy, 1994) qu'il s'agisse d'un titre, d'un nom de métier, d'un terme de parenté, d'un patronyme, d'un prénom, d'un terme affectif / dépréciatif, d'un déictique (l'emploi des pronoms reflétant la proximité / distance entre locuteurs (Kerbrat-Orecchioni, 1992)), de petits mots du 
discours (interjection, apostrophe...), mais aussi de tous les éléments qui sont d'ordre mimo-gestuel.

Comme nous allons le voir, les termes d'adresse occupent une place centrale dans la violence verbale du fait des différentes valeurs qu'ils sont susceptibles de prendre (Rosier, 2007): valeur déictique (identification marquée de l'interlocuteur), valeur interactionnelle (signalisation des tentatives de prises de parole), valeur relationnelle (adoucissement ou durcissement d'une requête de la déférence au mépris) et valeur de place (négociation des identités dans l'interaction). Ainsi, les termes d'adresse ont généralement, en plus de leur valeur déictique, une valeur relationnelle: lorsque plusieurs formes sont déictiquement équivalentes - comme «tu » et "vous » employés pour désigner un allocutaire unique -, elles servent en outre à établir un type particulier de lien social. La théorie «classique » de Brown et al. se résume comme suit (KerbratOrecchioni 1992, 17 et s.) : les systèmes d'adresse peuvent être ramenés à deux grandes catégories de formes qui sont la "distance» et le «statut / pouvoir». Leur usage réciproque reflète une relative égalité de statut entre les participants, et leur fonctionnement dissymétrique exprime une relation hiérarchique, particulièrement mobilisable dans la violence verbale.

Dans cette optique, les termes d'adresse, tout comme les honorifiques, démontrent qu'à travers ces usages interactionnels (pouvoir / familiarité) la compétence linguistique et la compétence socioculturelle ne sont que les deux faces d'une «compétence communicative " globale et subtile. Par exemple, l'emploi des pronoms personnels reflète bien la distanciation ou la proximité qui peuvent s'installer dans des situations pédagodidactico-scolaires et mobilisées selon les besoins interactionnels. Par exemple, l'emploi des déictiques de personne «nous» et «on» par un enseignant («on» équivalant préférentiellement à « nous » dans l'oral ordinaire) sont réducteurs de distance entre sa position dominante et la position dominée des élèves ; au contraire de l'emploi, toujours par l'enseignant, de « je / tu » pour interpeller un élève et " vous » pour interpeller le groupe classe qui sont producteurs de distance (le dominant tutoie le dominé qui le vouvoie). Ainsi, les termes d'adresse sont des marqueurs particulièrement évidents de la relation interpersonnelle et le pronom de deuxième personne peut constituer le procédé par excellence d'affichage de la distance (lorsqu'on lui oppose un "vous» non réciproque) ou de la familiarité voire de la solidarité (dans un emploi d'un «tu» réciproque).

6 Nous allons donc explorer, à travers des exemples, les effets de hiérarchie ou de familiarité non partagée sous-tendus par les termes d'adresse et qui peuvent produire de la violence verbale.

\section{De l'affront à la médiation : l'emploi détourné de « madame "}

7 À partir de nos observations nous nous sommes interrogées sur les liens qui existent entre la manière dont on s'adresse à l'interpellé et l'émergence de la violence verbale, telle du moins qu'elle est ressentie. Les analyses conduites ont porté sur les termes d'adresse comme « madame ", « monsieur ", les noms propres, et enfin les déictiques.

8 Nous avons ainsi pu observer que "madame», à la fois terme d'adresse et d'interpellation, présente un emploi détourné de durcisseur mais aussi un emploi médié 
d'adoucisseur en fonction des situations d'énonciation, auxquelles il est nécessaire de lier la subjectivité et le rôle social des interactants.

\subsection{L'ironie en milieu scolaire}

Dans le cadre d'une étude de terrain (Corpus Schultz-Romain, 2005) portant sur l'analyse d'interactions verbales en classes de $4^{\mathrm{e}}$ et de $3^{\mathrm{e}}$ (collège inscrit en Z.E.P. de la ville de Vitrolles, commune des Bouches-du-Rhône) (Romain, 2005, 2008), nous avons pu recueillir des interactions entre un enseignant et des élève(s) intervenant à l'occasion du cours de français.

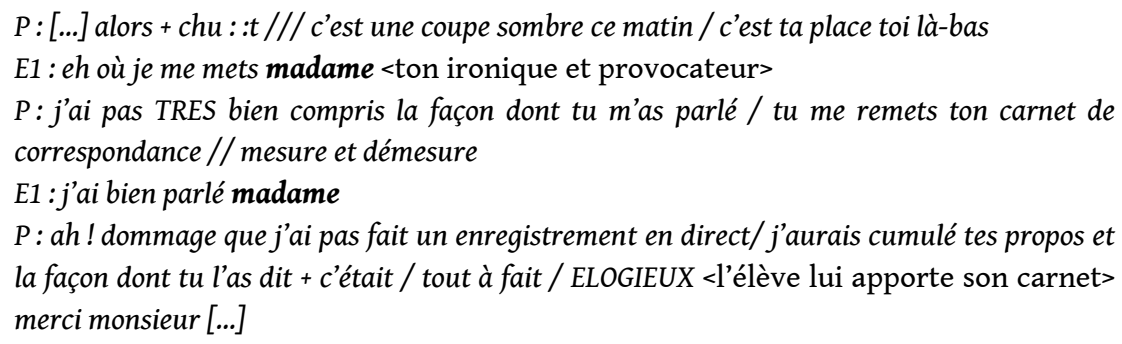

Dans cet exemple, le premier terme d'adresse "madame » prononcé par l'élève introduit une réponse globalement insolente, où il laisse entendre qu'il est assis à sa place et qu'il n'y a pas d'autre endroit où il pourrait s'installer, ce qui conduit l'enseignante à lui demander son carnet de correspondance. Dans cet exemple en particulier, il est intéressant de voir la différence d'adresse entre les deux "madame», le premier apparaissant dans un contexte où le ton, autant que le terme, ont visiblement suscité une interprétation de type "montée en tension", alors que le second "j'ai bien parlé madame » fait plutôt figure d'adoucisseur pour tenter de remédier au premier. Dans les deux cas, pourtant, l'élève utilise le cadre formel de la politesse pour s'exprimer, mais avec deux signifiés antithétiques.

\subsection{L'assénement du genre}

Dans un autre type de contexte, celui du débat politique médiatisé entre deux concurrents, on peut arguer que l'emploi, non seulement récurrent, mais martelé de « madame » (à la fois interpellatif et interruptif) comme dans le débat du 2 mai 2007 entre Nicolas Sarkozy et Ségolène Royal, contribue à rappeler constamment aux auditeurs le genre de l'adversaire et, ainsi, à le problématiser de facto dans le débat. C'est ce que nous avons appelé « l'attaque courtoise » (Fracchiolla, 2008). L'utilisation de la lexicométrie ( Lexico3, SYLED, Paris 3) nous a permis en particulier de calculer, à partir d'une retranscription exhaustive des formes complètes prononcées (nous n'avons pas, en effet, comptabilisé ici les formes commençantes ou avortées, comme l'ont fait De Chasnay et Kerbrat-Orecchioni dans l'étude qu'ils ont présentée au séminaire sur l'argumentation organisé par Marianne Doury, à Paris, le 12 juin 2008), 115 occurrences de «madame » prononcées par Nicolas Sarkozy contre seulement 7 « monsieur " prononcés par Ségolène Royal. Cette extrême déférence permet en effet au candidat masculin de demeurer dans un cadre de politesse et de courtoisie où coexistent un registre d'une extrême politesse comme "pardon », « excusez-moi » avec, au contraire, des actes de langage tout à fait offensifs sur fond de badinage courtois. Cette technique plus ou moins calculée a, dans tous les cas, comme effet le rappel constant qu'elle est une femme et lui un homme, ce qui 
vaut, à ce moment-là et dans cette énonciation particulière, rappel constant d'une faiblesse.

\subsection{Un emploi médié - adoucisseur - au supermarché et dans le tramway}

12 L'interaction suivante se déroule au supermarché. Elle est issue du corpus de Carole Viaud-Gayet (2003) constitué dans le cadre d'une Thèse de doctorat sur les conduites de la sociabilité ordinaire dans l'espace public, L'égard et la règle. Déboires et bonheurs de la politesse entre inconnus (Viaud-Gayet, 2008). La caissière interpelle une jeune femme qui perd patience parce qu'elle vient de se faire doubler dans la file par une femme d'environ 65 ou 70 ans, qui, ce faisant, ne lui a adressé ni un mot ni un regard:

- Hé oui / mais franchement madame / vous croyez que ça vaut la peine de s'énerver pour ça / Laissez courir va / c'est une vieille dame, on sait pas nous comment on sera à cet âge-là.

Dans un autre exemple, toujours issu du même corpus, une cliente s'adresse à une autre cliente. La première s'insurge de ce qu'une troisième femme, arrivée en dernier, s'est littéralement précipitée pour prendre la première place à une nouvelle caisse venant de s'ouvrir, sans considération pour ceux qui attendaient, avant elle, à côté :

- Laissez va / madame, vous vous en fichez, qu'est ce que ça peut faire /

Enfin, un dernier exemple de scène interpellative en espace public se déroule dans le tram de Montpellier. Ce corpus a été recueilli sur le vif, lors d'un déplacement « étude de terrain » pour observer la médiation des contrôleurs dans les transports publics (Corpus Auger, 2007).

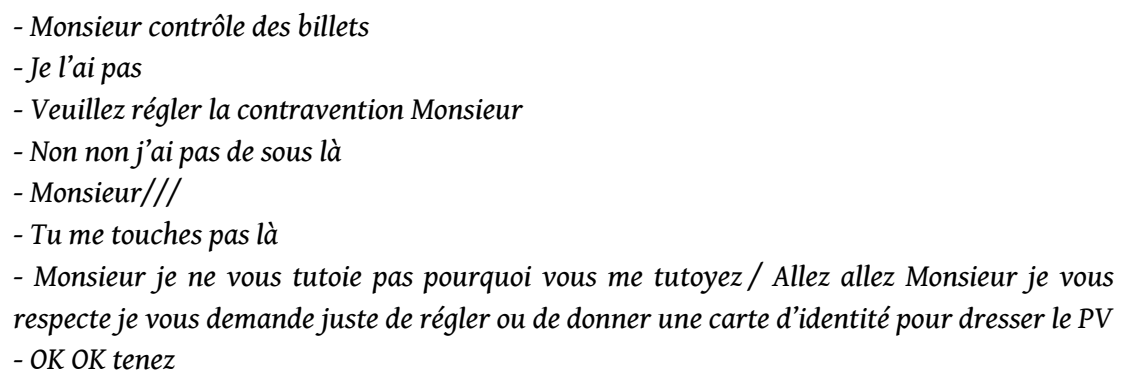

Ici le «monsieur» interpellatif répété avec constance sert à la fois de marqueur d'autorité et d'adoucisseur dans la mesure où il maintient la distance polie, qui équivaut également au respect de l'interlocuteur. L'effet est particulièrement perceptible dans l'échange, du fait du tutoiement du passager, ressenti et présenté comme un manque de respect, une prise de pouvoir sur l'autre (et non comme un effet de connivence possible) et donc une violence. On est face à une situation où la politesse est rendue tant par un comportement que par un code approprié visant à montrer le respect. Ce code fonctionne puisque l'usager cède non pas à l'autorité, mais à la nécessité marquée de respecter la personne qu'il a en face de lui. Ici, d'une certaine façon, on peut comprendre que c'est la relation intersubjective qui l'emporte sur la relation d'autorité entre contrôleur et usager. Le représentant de l'autorité sert en réalité de médiateur. 


\section{Entre durcisseurs ou adoucisseurs : noms propres, appellatifs et déictiques}

16 Aux côtés des différents «madame» et "monsieur», les titres, noms propres et appellatifs peuvent également occuper une place de durcisseur.

\subsection{Le CPE, noms propres et effets de catégorisation}

17 Lors de la crise du CPE, nous avons recueilli divers corpus de ce type avec effets de catégorisation par des surnoms (Corpus Fracchiolla et Moïse, 2006), aussi bien dans le discours rapporté par la presse que dans les manifestations elles-mêmes (en particulier, à Marseille, Paris et Montpellier). On trouve ainsi Chirac - alors président de la République - représenté et désigné comme le bouffon, le menteur et comme "Super menteur». Parallèlement, Sarkozy (alors ministre de l'intérieur) est désigné et représenté sous la forme du super anti-héros méchant avec des appellatifs tels que "Karsherator » et « Darkvador » qui renvoient l'un indirectement par suffixation et métaphore au Karsher brandi comme une arme, l'autre directement, au méchant héros de la Guerre des étoiles, représentant «le côté obscur de la force » et alors très présent aux esprits puisque le dernier épisode vient de sortir. À côté se trouvent également des désignations de D. de Villepin qui font allusion à l'aristocrate, au tyran tout en exploitant les mêmes sonorités allusives renvoyant au héros de la Guerre des étoiles Darkvador : "Vilpintator » (avec en plus un jeu de mots sur Villepin t'as tort). D'autres pancartes encore présentent Chirac comme le «bouffon », soit de manière visuelle (marionnette avec un nez rouge), soit par désignation directe (Fracchiolla et Moïse, à paraître).

\subsection{Les déictiques dans le débat politique}

On remarque enfin également une utilisation particulière des pronoms, qui peut renvoyer elle aussi au syndrome de l'ultra-politesse, comme cela est le cas dans le débat entre Nicolas Sarkozy et Ségolène Royal du 2 mai 2007, déjà évoqué. On relève en effet dans ce débat une différence de style entre les deux candidats par rapport au mode d'adresse. Alors que Ségolène Royal utilise « je » et « ils / elles » afin d'établir un lien entre « elle » et " eux ", Nicolas Sarkozy parle en revanche de manière plus impersonnelle, détachée, en ayant recours aux « on » et "vous » tout en s'adressant à sa partenaire de débat de façon très directe. Par ailleurs, si les deux candidats s'adressent l'un à l'autre à l'aide de la formulation "vous" associée à un verbe conjugué de manière très équilibrée, on remarque que Nicolas Sarkozy utilise le « vous » tout seul comme terme d'adresse poli 39 fois de plus que Ségolène Royal, ce qui renforce le phénomène d'interpellation par « madame » précédemment signalé. À savoir que Nicolas Sarkozy fait un usage tout à fait particulier de la politesse dans la mesure où c'est le fait même de rester dans le cadre de la politesse qui lui permet d'être plus agressif qu'il ne serait normalement autorisé à l'être socialement à l'égard d'une femme. Il s'agit donc ici d'une utilisation que l'on peut comprendre comme déviante ou détournée des formes «madame » et "vous », car elles donnent l'illusion de la déférence, alors que le contenu des propos qu'elles entourent est au contraire chargé de piques et de montées en tension. Contenant toutes les formes de la 
politesse, cela rend alors en effet le discours de Nicolas Sarkozy acceptable à tous égards aux citoyens auditeurs.

\subsection{Les déictiques dans l'interaction pédago-didactique}

19 Dans le cadre de l'étude de terrain précédemment évoquée portant sur l'analyse d'interactions verbales au collège (Romain, 2005, 2008), nous avons pu recueillir des interactions entre enseignant et élève(s) intervenant à l'occasion de cours de français dans deux collèges appartenant à des milieux socio-culturellement contrastés. Nous avons pu observer que conformément à la relation complémentaire classique, la position haute des enseignantes en milieu dit favorisé détermine la relation dissymétrique qui unit enseignants et élèves et repose sur une stricte distance institutionnelle. Cette position haute dominante est entretenue par le recours à des outils linguistiques que sont les déictiques «je», «tu» et "vous», renforcée par le recours à l'impératif (ellipse du pronom personnel d'adresse) et aux prénoms des élèves dont voici deux exemples :

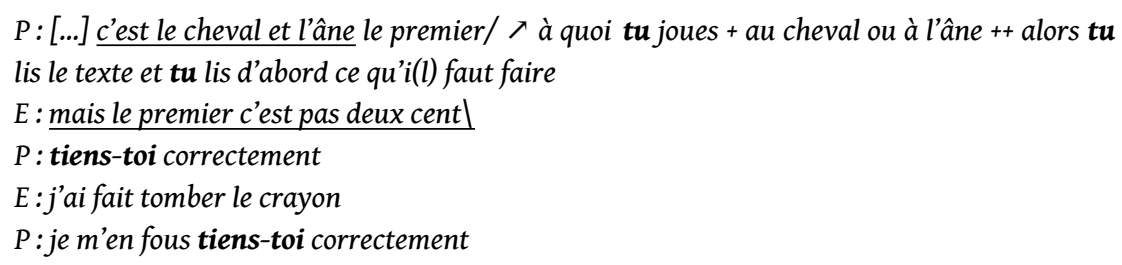

Ce mode de relation interpersonnelle pédagogique est divergent avec celui recueilli en milieu dit défavorisé, où la position haute est peu sollicitée et, de fait, ne crée pas de distance fortement dissymétrique entre enseignantes et élèves. Ainsi, face à des adolescents difficiles les déictiques de personne sont moins consensuels et peinent à s'imposer, comme l'exemple suivant le montre :

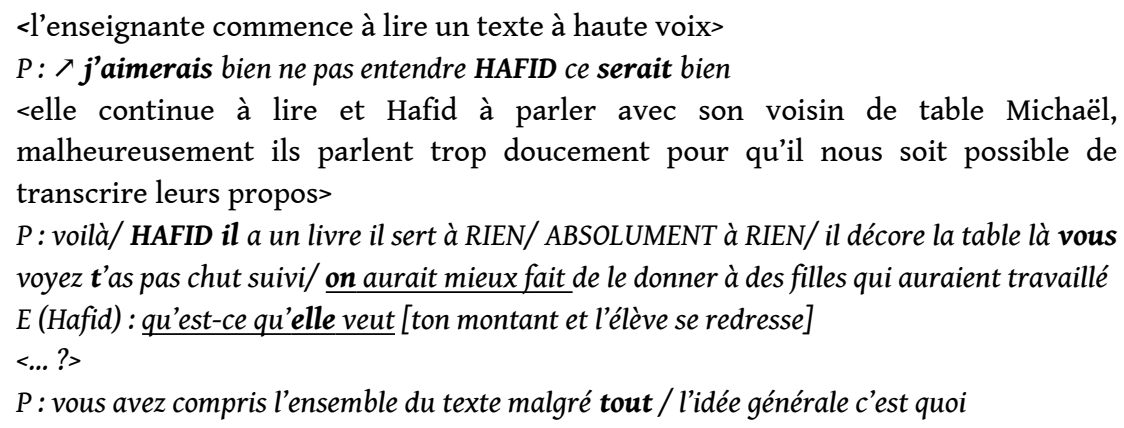

21 Ainsi à travers cet exemple, on observe que l'enseignante réprimande l'élève en s'adressant à lui à la troisième personne du singulier ; elle semble donc prendre la classe à témoin, en recourant à une formulation de type «Hafid il». Cette attitude lui évite une confrontation en face à face avec l'élève concerné. Lorsqu'elle recourt finalement au pronom d'allocution de la deuxième personne du singulier, elle le fait immédiatement précéder d'une prise à témoin, cette fois-ci tout à fait explicite, des autres élèves " vous voyez ", qu'elle inclut finalement dans sa propre énonciation par le recours au déictique «on » + conditionnel passé (forme composée) « on aurait mieux fait de le donner à des filles qui auraient travaillé ». Le conditionnel passé a lui aussi un effet modéré sur le discours de l'enseignante : non seulement elle joue de la connivence avec sa classe mais l'emploi du conditionnel passé semble servir d'atténuation à l'énoncé directif de l'enseignante, contrairement à une réalisation qui serait faite au présent. Dans le même temps, l'élève concerné par la réprimande chevauche de ses propos l'intervention de 
l'enseignante d'un «qu'est-ce qu'elle veut». Il s'agit moins d'une question que d'une démarche réactive, une réplique à l'encontre de l'enseignante. Il s'agit donc d'un acte directif ardent qui cherche à faire fléchir l'enseignante, à l'intimider et à la provoquer mais aussi à masquer l'atteinte qu'il a subie - puisque l'enseignante est en train de le réprimander et qu'elle a pris à partie la classe. Par conséquent l'élève réagit par une agression verbale à l'encontre de l'enseignante. Cette agression est renforcée par le « elle », qui a une valeur dépréciative, par lequel l'élève présuppose que l'enseignante se mêle de ce qui ne la regarde pas et que son intervention a un caractère infondé. Il tente ainsi de la faire taire.

L'enseignante ne va pas réagir à cet acte offensant pour sa personne et va donc rester en position basse. L'enseignante ne réprimande pas cette dernière intervention de l'élève réprimandé qui, d'une part l'offense par son caractère ostensiblement impositif, et d'autre part méconnaît une règle d'organisation des tours de parole dans la classe, qui ne peut être vécue autrement que comme un affront vis-à-vis d'elle-même. En n'émettant aucune réaction, elle fait preuve de politesse négative. On peut penser cette attitude abstentionniste comme une stratégie lui permettant de poursuivre son cours et d'éviter le conflit avec un élève - ce qui pourrait la conduire à perdre plus durablement sa position haute. Bien qu'elle n'évite pas pour autant l'affront, qui atteint sa face, elle évite d'y répondre et de risquer une montée en tension. Notons cependant la présence du « malgré tout » qui permet à l'enseignante de revenir implicitement sur ce qui vient d'être dit, et finalement d'atténuer la valeur perturbatrice de l'énoncé de l'élève. En ne donnant pas de place centrale à cet énoncé (qui susciterait une interruption du cours), elle diminue son impact ; ce qui lui permet de reprendre la direction du dialogue interdiscursif.

Cette différence concernant le recours aux termes d'adresse et appellatifs souligne donc un mode de relation interpersonnelle ou, à tout le moins, un mode de choix énonciatif différent suivant la population d'élèves considérée (mais aussi et nécessairement suivant les enseignantes considérées). Autrement dit, on assiste en milieu difficile à une relation interpersonnelle bien plus coopérative qu'elle ne l'est en milieu dit favorisé avec un recours préférentiel aux déictiques «nous" et "on", ainsi qu'à l'utilisation du conditionnel; tandis qu'au contraire, on assiste en milieu facile à une relation interpersonnelle nettement plus agonale avec un recours préférentiel aux déictiques «je », «tu», "vous », ainsi qu'à l'interpellation des élèves par leur prénom et par le recours à l'impératif. Nous avons donc pu observer que, par rapport à l'acte de langage produit par l'enseignante, les termes d'adresse servent à adoucir (pour les enseignantes du collège difficile) ou à durcir (pour les enseignantes du collège facile) une intervention en fonction du registre discursif de l'enseignante considérée : adoucissement pour le registre réducteur de distance institutionnelle pour les enseignantes du collège dit difficile et durcissement pour le registre discursif plus agonal du collège dit facile, entretenant ainsi la distance enseignante / élève(s). Nous pouvons donc souligner le fait que les termes d'adresse ont un rôle certain dans la négociation des identités et de la relation interpersonnelle. 


\section{Conclusion : le rôle pivot de l'interpellation dans la montée en tension de la violence verbale}

24 À partir de ces analyses, nous pouvons dire que l'interpellation tient une place importante dans la violence verbale. Non seulement parce qu'elle peut produire des effets " violents », par la façon dont l'individu est désigné, interpellé ou apostrophé, mais aussi, parce qu'elle peut apparaître à toutes les étapes de la montée en tension.

Si elle est à relier au contexte général de communication comme forme de climat général (Galatalo et Mizza, 1998), la violence verbale se subdivise en quatre étapes distinctes qui aboutissent à un schéma typique de violence. Cependant, à chacune de ces étapes, des phénomènes de réparation peuvent intervenir et désamorcer cette même montée en tension, ce qui est le cas par exemple dans l'exemple du tramway.

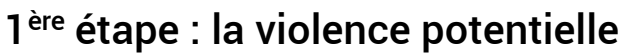

La violence potentielle est liée à la personne elle-même, à son agressivité comportementale, ou à un contexte supposé violent qui est lui-même construit à travers des représentations ou mises en scène intersubjectives ou médiatiques, comme les images renvoyées par les médias des banlieues. Dans nos corpus, les lieux - en particulier publics et institutionnels - susceptibles de générer cette violence sont les contrôles dans les transports, les grèves, les débats politiques, l'attente aux caisses de supermarché, les salles de classe... Les personnes supposées potentiellement «fauteuses de trouble » sont les usagers, grévistes/manifestants, élèves (ceux qui endossent un statut asymétrique) susceptibles d'entrer dans des échanges interpellatifs pour protester, demander, s'insurger contre une situation ressentie comme violente.

Dans le cadre d'une enquête menée dans un collège d'éducation prioritaire de Perpignan (Corpus Fillol et Moïse, 2003), nous avons pu assister à une montée en tension violente entre des élèves et une enseignante de français (Auger, Moïse et alii, 2003). Avant d'en arriver à l'altercation même, l'enseignante avait été interpellée à maintes reprises par les élèves, signes déjà d'un cadre instable, fait de tensions vécues ou à venir. L'enregistrement commence au début du cours. Plusieurs élèves, dont Fouad, cherchent à obtenir l'attention de l'enseignante. En vain.

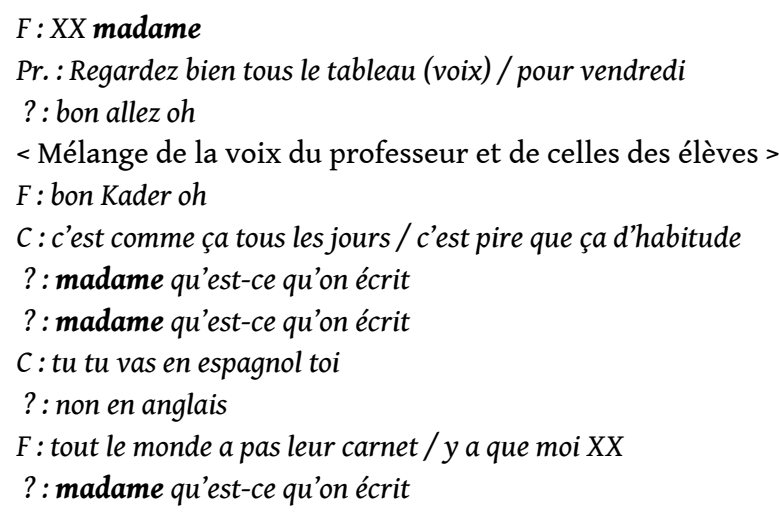

Dans cet extrait, les élèves demandent une prise de parole ou une réponse qui ne viennent pas. Cet échec d'une demande d'interactions va provoquer par la suite le déclenchement d'une altercation violente. Les interpellations se voient vouées à l'échec ; 
elles entretiennent alors un climat général de tension et sont perçues par les élèves comme une position d'autorité de la part de l'enseignante. Ainsi, par la suite, face à la demande de l'enseignante de présenter son carnet de liaison, un des élèves, Kader, tente d'expliquer qu'il lui a été retiré lors du cours précédent. Malgré une apparente coopération de la part de l'élève, dans le contexte de la classe, son argumentation n'est pas entendue et il hausse le ton pour entrer dans une opposition caractérisée.

\section{$2^{\text {ème }}$ étape : la violence embryonnaire}

L'amorce de la violence verbale est «lancée » par un locuteur A (interpellation) et va entrainer certains modes de réactions de la part du locuteur B. Dans nos corpus nous avons recensé comme indicateurs les termes d'adresse comme " madame", ironique en salle de classe ou répétitif (créateur de harcèlement dans le débat politique); les déictiques "vous / tu» (voir l'exemple du tramway), ou encore l'agressivité avec changement prosodique et posture particulière du corps (exemple du tramway à nouveau);

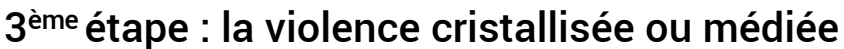

La montée en tension se joue et se rejoue dans les différentes prises de parole des locuteurs sous forme de boucles interactionnelles ou A et B interéchangent leur place au cours d'une joute verbale (comme dans l'exemple de la classe). Cette entrée marquée dans la violence verbale peut être dépassée ou évitée. Le locuteur B ou un tiers peut tenter une négociation qui porte sur l'objet du conflit, comme dans le corpus Viaud-Gayet ou sur la relation interpersonnelle, comme dans l'exemple $\mathrm{du}$ tram. Toutes ces stratégies permettent une résolution non violente du conflit et aplanissent à chaque fois une situation où les modes interpellatifs ont contribué à l'éclosion d'une montée en tension violente.

31 Face aux attaques de A, le locuteur B peut adopter différents comportements. Il peut notamment entrer résolument dans le conflit et prendre part à la montée en tension en répondant par exemple à l'interpellation par l'interpellation : «merci Monsieur » répond l'enseignante au collégien dans la salle de classe; ou au contraire chercher la médiation (exemple du tramway). Dans les deux exemples précédemment cités (corpus Viaud-Gayet et tramway), on voit comment le recours à un code formel de politesse, identifié et reconnu comme tel, aide à structurer le dialogue vers une issue médiée. Ici, il n'y a pas besoin de médiateur extérieur, car c'est la mise en discours qui joue ce rôle et redistribue les places. La politesse donne un cadre rassurant d'interaction comme d'interpellation, où personne n'est menacé et où l'échange peut donc avoir lieu à nouveau de manière confiante.

32 Ainsi, les effets violents de l'interpellation et du propos adressé peuvent être dépassés ou évités à travers d'autres résolutions conversationnelles. Le locuteur B peut tenter de mettre un terme au conflit par la négociation, qui portera sur l'objet même du conflit ou sur la relation interpersonnelle. Dans ce cas, il faut que les deux locuteurs soient capables de « s'entendre » hors de tout sentiment d'atteinte à la face (Goffman, 1973). D'une autre façon, le locuteur B peut décider d'opter pour la fuite ou l'évitement. La fuite consiste à se taire, voire à physiquement partir, ou encore à opter pour un changement thématique (« bon parlons d'autre chose »). L'évitement consiste, lui, à rester dans la thématique sans 
contre-attaquer: les marques d'humour peuvent en être une manifestation. Ainsi, certains modes d'interpellation peuvent parfois, selon les contextes, se charger de violence et aller jusqu'à l'insulte. Ce peut-être le cas, entre autres et comme nous l'avons $\mathrm{vu}$, de l'emploi des termes d'adresse «madame » ou «monsieur, » ou encore du pronom d'adresse tu.

\section{$4^{\text {ème }}$ étape : la violence physique}

L'insulte est souvent le point de rupture avant la violence physique mais, au-delà de la violence verbale, l'ultime recours pour se faire entendre quand rien d'autre n'a fonctionné est la violence physique dans une forme de passage à l'acte souvent annoncé pragmatiquement par la parole (sur le mode «toi, je vais te casser la gueule») ou le mimo-gestuel dans les montées en tension, avec, encore une fois, usage de l'interpellation. Par exemple dans un extrait du film Karnaval de Thomas Vincent que nous avons étudié (Auger et Moïse, 2005), un fils interpelle son père et insulte de façon indirecte son frère (« Nasser / il te lèche le cul / Nasser c'est tout »), ce qui va enclencher en réaction le passage à la violence physique.

Pour finir, qu'elle soit marque de négociation ou d'entrée dans le conflit, l'interpellation joue un rôle clé dans la violence verbale. Ce que, dans sa perspective, avait souligné Louis Althusser $(1970,125)$ en faisant le lien entre interpellation et idéologie. L'interpellation quotidienne pouvait prendre le poids du pouvoir dominant quand, par exemple, le " hé vous là-bas " renvoyait à une interpellation policière, marque possible d'un déclenchement d'une montée en tension. Comme quoi, l'interpellation pouvait se charger d'une force bien plus grande que celle des rituels quotidiens...

\section{BIBLIOGRAPHIE}

ALTHUSSER L., 1970, « Idéologie et appareils idéologiques d'Etat », in Positions, Paris, Ed. Sociales ANDRÉ-LAROCHEBOUVY, D., 1994, Introduction à l'analyse sémio-linguistique des conversations, Paris, Credif-Didier.

AUGER, N., MOÏSE, C. et alii, 2003, « La violence verbale : enjeux, méthode, éthique », Castellotti, V. et D. de Robillard (Éds) France, pays de contacts de langues, Actes du colloque de Tours, 9-10 novembre 2000, Cahiers de l'institut de linguistique de Louvain, numéro 29, volume II, Louvain-LaNeuve : 131-150.

AUGER N., 2003, « Dimensions identitaires des participants aux interactions orales en classe : le cas d'une classe ZEP d'un collège de Perpignan » in Les didactiques de l'oral, actes du Colloque de l'université Montpellier III, IUFM de Montpellier, 14 juin 2002 : 185-196.

AUGER N. et MOÏSE C., 2005, « Violence verbale, malentendu ou mésentente ? », Bacha, G., Laroux, G. et Séoud, A. (Eds), Actes du colloque de Sousse (Tunisie), 15-17 avril 2004, Le malentendu, Université de Sousse, Presses Internationales de la Faculté des Lettres de Sousse, Tunisie : 293-302. 
FRACCHIOLLA B., et MOÏSE C., (à paraître) « Construction de la violence verbale et circulation des discours : autour du contrat première embauche », colloque Ci-dit, université de Laval, Québec, Circulation des discours et liens sociaux : le discours rapporté comme pratique sociale, 7-9 octobre 2006, Montréal, Éditions Nota bene.

FRACCHIOLLA B., 2008, «L'attaque courtoise : de l'usage de la politesse comme stratégie d'agression dans le débat Royal-Sarkozy du 02 mai 2007 », Actes des JADT 2008, Presses Universitaires de Lyon : 495-508.

GALATALO, R. et MIZZAU, M., 1998, « Conflit conversationnel et malentendu : quelques relations possibles », La linguistique 34-1 : 151-164.

GOFFMAN E., 1973, La mise en scène de la vie quotidienne, Paris, Ed. de minuit, 2 tomes.

GOFFMAN E., 1974 (trad. fr.), Les rites d'interaction, Paris, Ed. de Minuit.

KERBRAT-ORRECCHIONI, C., 1990, Les interactions verbales, 3 tomes, Paris, Armand Colin. Tome 2. 1992.

MOÏSE C., 2004, « Postures sociales, violence verbale et difficile médiation », Les médiations langagières (Dir. Delamotte-Legrand), Actes du colloque de Rouen, 7-8 décembre 2000, Presses de l'université de Rouen : 335-349.

MOÏSE C. 2006, « Analyse de la violence verbale : quelques principes méthodologiques », actes des $\mathrm{XXVI}^{\mathrm{e}}$ Journées d'étude sur la parole, 12-16 juin, Dinard, http://jep2006.irisa.fr/ JEP06_ACTES.PDF

MOÏSE C., AUGER N., FRACCHIOLLA B et SCHULTZ-ROMAIN C. (éds), 2008, De l'impolitesse à la violence verbale, deux tomes, Paris, L'Harmattan, coll. Espaces discursifs.

SCHEGLOFF, E.A., 1979, « Identification and Recognition in telephone openings », in G. Psathas (ed), Everyday Language, New York, Erlbaum, 23-78.

ROMAIN C., 2005, La gestion discursive de la relation interpersonnelle dans la classe de français, Collection « Sémantiques », Paris, L'Harmattan.

ROMAIN C, 2008 : « Description de la violence verbale en situation difficile d'enseignement », Actes du colloque international «De l'impolitesse à la violence verbale », 2005, Centre de recherche interdisciplinaire, langue et identité culturelle, Université d'Avignon, collection Espaces discursifs, Paris, L'Harmattan.

ROSIER L., 2007, Petit traité de l'insulte, Bruxelles, Edition Labor.

VIAUD-GAYET, C., 2008, « Les disputes de politesse dans l'espace urbain : quand la politesse tourne à la violence ", in Moïse, C., Auger, N., Fracchiolla, B., Schultz-Romain, C., De l'impolitesse à la violence verbale, tome 1., Espaces politiques et médiatiques, L'Harmattan : 147-183.

\section{ANNEXES}

\section{Convention de transcription}

Les pauses, selon leur durée, sont marqués par /, ou //, ou encore ///.

Un mot incompréhensible se note par (X), un passage plus long par (XXX), une incertitude de transcription par (de X). 
L'allongement est noté par :

(rires) est un commentaire d'un comportement non verbal.

L'hésitation entre deux formes, bien souvent morphologiques, est citée entre parenthèses (j'ai été / j'étais) (i regarde(nt) (ces / ses). Cette hésitation peut se manifester aussi entre la forme pleine et sa non manifestation (ça a été / ça ø été)

Les paroles simultanées sont soulignées

Les liaisons non conformes à la norme sont marquées avec trait d'union, j'suis-t-allé. Le n' de liaison ou de négation est marqué entre parenthèse, on (n') y était pas .

\section{RÉSUMÉS}

Notre contribution traite de l'interpellation et de ses implications socio-pragmatiques dans les cas de violences verbales. Notre corpus s'est construit selon une méthodologie ethnographique à travers plusieurs terrains (espaces publics - rue, transports en commun-, établissements scolaires, médias etc.), développée grâce à un travail d'équipe depuis 2001. Les données recueillies ont été ensuite étudiées sous forme d'analyses conversationnelle et de discours. Nous nous attachons ainsi dans cet article aux termes d'adresse ou appellatifs recensés dans nos corpus de violence verbale (débat politique, école, guichet, rue). Si l'interpellation «s'inscrit dans l'improvisation de l'interlocution » selon l'argument du colloque, comment cet élément verbal peut-il être déclencheur ou non d'interactions verbales agonales? Nous analyserons les types d'interpellations que nous pouvons recueillir, qui rendent compte de différents types d'opérations et de catégorisations et qui engendrent violences verbales et physiques (effets sociopragmatiques de l'interpellation). Nous associons l'étude de l'interpellation à « la manière dont l'interpellé est désigné » mais aussi à « la structuration du propos qui lui est adressé » (recipient designed selon Schegloff 1979), quand ces éléments sont éclairants pour l'analyse.

The proposed article focuses on «interpellation » as sociolinguistic and pragmatic meanings in verbal violence. This study situates itself within the field of sociolinguistics and follows an ethnographic methodology. It has concentrated on a lot of places like public transport system, on the street, at school, on the radio, etc. This includes the collection of different types of empirical data: interviews, participant observation, written documentation or authentic interactions. Then, our analysis aims at understanding the verbal violence process through conversational and discourse methodology. Therefore from our field work we will here explore how interpellation can set off and release (or not) verbal violence in interactions.

INDEX

Mots-clés : interpellation, violence verbale, montée en tension, nomination, catégorisation

\section{AUTEURS}

\section{NATHALIE AUGER}

Laboratoire Dipralang, EA 739, Université de Montpellier 3 
BÉATRICE FRACCHIOLLA

Laboratoire SYLED-EA2290, Université de Paris 3, Université de Paris 8 \& MSH Paris Nord

CLAUDINE MOÏSE

Laboratoire ICCT, EA. Université d'Avignon

CHRISTINA SCHULTZ-ROMAIN

Laboratoire LPL, IUFM d'Aix 